\title{
Requirements for acquiring a high-quality house dust mite extract for allergen immunotherapy
}

This article was published in the following Dove Press journal:

Drug Design, Development and Therapy

16 May 2012

Number of times this article has been viewed

Franco Frati'

Cristoforo Incorvaia ${ }^{2}$

Marie David ${ }^{3}$

Silvia Scurati ${ }^{3}$

Simona Seta ${ }^{4}$

Guglielmo Padua ${ }^{4}$

Eleonora Cattaneo'

Carlo Cavaliere ${ }^{5}$

Alessia Di Rienzo 6

Ilaria Dell'Albani'

Paola Puccinelli'

'Medical and Scientific and Regulatory Department, Stallergenes, Milan, Italy; ${ }^{2}$ Allergy/Pulmonary Rehabilitation, ICP Hospital, Milan, Italy; ${ }^{3}$ Laboratoire Stallergenes, Antony, France; ${ }^{4}$ Marketing Department, Stallergenes, Milan, Italy; ${ }^{5} \mathrm{Ear}$ Nose and Throat Department, University Sapienza, Rome, Italy; ${ }^{6}$ Azienda Sanitaria Locale, Allergology Service, Frosinone, Italy

\section{Video abstract}

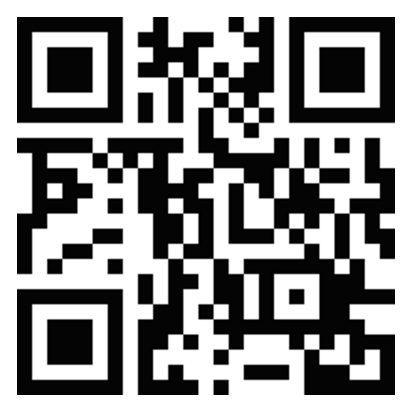

Point your SmartPhone at the code above. If you have a $Q R$ code reader the video abstract will appear. Or use: http://dvpr.es/HWp29T

Correspondence: Cristoforo Incorvaia Allergy-Pulmonary Rehabilitation ICP Hospital,Via Bignami I, 20126 Milan, Italy Tel +39025799 3289

Fax +390257993315

Email cristoforo.incorvaia@gmail.com
Abstract: The house dust mite is a major cause of respiratory allergy worldwide. The management of mite allergy is based on avoidance measures, drug treatment, and allergen immunotherapy, but only allergen immunotherapy is able to modify the natural history of the disease. Injectable subcutaneous immunotherapy was introduced a century ago, while sublingual immunotherapy was proposed in the 1980s and emerged in the ensuing years as an effective and safe option to subcutaneous immunotherapy. However, the quality of the extracts to be used in allergen immunotherapy is crucial for the success of treatment. The mite extract for sublingual immunotherapy known as Staloral 300 was developed to offer optimal characteristics concerning the mite culture medium, standardization, and allergen dose. Double-blind, placebo-controlled trials with Staloral 300 have provided a substantial part of the clinical evidence analyzed in a meta-analysis of the efficacy of allergen immunotherapy in mite-induced rhinitis and asthma. Safety and tolerability are very good, mild local reactions in the mouth being the most common side effect. This makes it feasible to carry out sublingual immunotherapy for the $3-5$-year duration needed to achieve long-lasting tolerance to the specific allergen. The performance of Staloral 300 may provide optimal conditions for an effective and safe sublingual immunotherapy in patients with mite-induced respiratory allergy.

Keywords: house dust mite, rhinitis, asthma, allergen extracts, sublingual immunotherapy

\section{Introduction}

The house dust mite is a major cause of respiratory allergy worldwide. ${ }^{1-3}$ Mites are taxonomically classified into more than 50,000 species, but those belonging to the subfamily of Pyroglyphidae, and particularly the species Dermatophagoides pteronyssinus and Dermatophagoides farinae, are particularly involved in allergic diseases. ${ }^{4} \mathrm{D}$. pteronyssinus and $D$. farinae generally coexist, humidity being the main factor favoring the former. ${ }^{2} \mathrm{~A}$ large number of allergens, comprising a number of different groups, has been identified in Dermatophagoides spp, ie, groups 1-11, 13 and 14, and 15-18, are present in both species, while group 20 is specific for D. pteronyssinus. ${ }^{5,6}$ In particular, group 1 and 2 include the major allergens Der p 1/Der f 1 and Der p 2/Der f2, respectively, that are recognized by IgE antibodies in more than $70 \%$ of mite-allergic patients. ${ }^{7}$ Both Der $p$ 1/Der f 1 and Der p 2/Der f 2 are generally found within mite fecal pellets, and reach high concentrations in indoor environments. ${ }^{7,8}$ Other clinically important allergens comprise groups $5,7,8$, and $10,{ }^{9}$ the latter including tropomyosin, which accounts for cross-reactivity with crustaceans and molluscs and thus for allergic reactions to foods. ${ }^{10}$ Two studies have systematically examined the amount of IgE binding to the different dust mite allergens. ${ }^{11,12}$ Der $\mathrm{p}$ 1/Der $\mathrm{f} 1$ are proteolytic enzymes with optimal characteristics to act as allergens, ${ }^{13}$ but also show biological activity favoring allergic 
sensitization. In particular, Der $\mathrm{p} 1$ affects tolerance through downregulation of indoleamine 2-3-dioxygenase expression in dendritic cells; stimulates the production of interleukin (IL)-4; decreases interferon- $\gamma$; degrades airway antiprotease-based lung defenses, leading to tissue damage; digests intercellular tight junction proteins, increasing the permeability of epithelium; directly activates airway epithelial cells, promoting production of proinflammatory cytokines, such as interleukin-6 (IL-6), IL-8, and granulocyte-macrophage colony-stimulating factor; activates human myeloid and plasmacytoid dendritic cells to initiate Th 2 cell responses; and directly elicits changes in airway smooth-muscle responsiveness. ${ }^{14-16}$ Moreover, the protease activity of Der $\mathrm{p}$ 1/Der $\mathrm{f} 1$ favors their facilitated entry through the skin and capture by dendritic cells ${ }^{17}$ that activate an initially local Th2 but a later Th1 response, along with a systemic Th2 response inducing isotype switching to IgE synthesis and involvement of eosinophils. ${ }^{18}$ Of note, Dermatophagoides spp are the only source of allergens with a protease being the major specificity. The ongoing inflammatory process is then sustained by Th2-related cytokines, such as tumor necrosis factor- $\alpha$, IL-5, IL-13, IL-17, and IL-31, the latter being primarily expressed in skin-homing Th2 cells. ${ }^{19}$ This, together with impaired function of filaggrin, which is a structural protein associated with filaments which are bound to keratin fibers in epidermal cells and ensures the barrier function to skin, is likely to account for the natural history of allergy to the house dust mite..$^{20}$

The treatment strategies to counteract this series of events are drug therapy, and especially topical and inhaled corticosteroids (but cannot act once treatment is stopped), ${ }^{21}$ allergen avoidance that is unable to reduce house dust mite concentration to less than the sensitization threshold level, ${ }^{22}$ and allergen immunotherapy. Allergen immunotherapy was introduced 100 years ago and showed constant evolution, also based on the improvement of quality of allergen extracts. ${ }^{23}$ Currently, there are two routes of administration of allergen extract, ie, subcutaneous immunotherapy and sublingual immunotherapy. This paper deals with the development of a pharmaceutical grade allergen extract of $D$. pteronyssinus and $D$. farinae, which represents a high-quality standardized product by which the performance of allergen immunotherapy can be improved.

\section{Obtaining a high-quality mite allergen extract}

The quality of an allergen extract is related to the adequacy of the original material and to standardization, enabling reproducibility of allergenic potency in extracts from different batches. Concerning the original material, unlike pollens, mites cannot be derived from naturally occurring sources but require cultivation in the laboratory, where the culture medium used is of pivotal importance.

\section{Culture medium}

The house dust mite feeds on human skin scales (giving the name Dermatophagoides after the Latin meaning "eating the skin") present in beds, upholstery, and carpets. ${ }^{24}$ However, material of human or animal origin must not be used for safety reasons in the culture medium used in the laboratory. Also, an optimal culture medium must allow growth of house dust mite expressing all major allergens and exclude any nonmite allergens. In the Stallergenes laboratory (Antony, France) an optimal culture medium (Stalmite APF [animal protein-free]) resembling the composition of the human stratum corneum and based on wheat germ, yeast, and amino acids in defined proportions of $42 \%, 42 \%$, and $15 \% \mathrm{w} / \mathrm{w}$, respectively, was developed. A proteomic analysis showed that D. pteronyssinus extracts obtained in such a state did not contain allergens developing from culture medium components and that the major group 1 and 2 allergens were demonstrated to be present as well as the allergens from groups 7, 10, 13, and 20. Groups 13 and 20 appear to be of little importance. ${ }^{25}$

\section{Standardization}

The process of standardization is based on: collection and selection of raw material from dust mites cultivated on Stalmite; macroscopic and microscopic identification from raw material; comparison of the protein profile with an in-house reference by isoelectric focusing and sodium dodecyl sulfate polyacrylamide gel electrophoresis; measurement of the allergen activity in comparison with the in-house reference by enzyme-linked immunosorbent assay inhibition; dosage of the major allergens, ie, the allergens recognized by more than $50 \%$ of patients allergic to the house dust mite; ${ }^{26}$ extraction in optimized conditions according to Good Manufacture Practice; clarifying filtration with elimination of extractive salts and low molecular weight substances; final adjustment of dosage of the allergen activity in comparison with the in-house reference; and measurement of the biological potency of the extract using the index of reactivity (IR).

The IR is a biological unit, and a concentration of 100 IR is defined by the capacity of the allergen to elicit by skin prick test a geometric mean wheal size of $7 \mathrm{~mm}$ diameter in 30 patients sensitive to the corresponding allergen. For the house dust mite, 100 IR corresponds to $40-70 \mu \mathrm{g}$ of 
mite proteins..$^{25}$ During the standardization procedure, the material is in the liquid phase. The product must be stored between $2^{\circ} \mathrm{C}$ and $8^{\circ} \mathrm{C}$. In this condition, the extract is stable for 12 months. At room temperature, the extract is stable for 3 months.

\section{Practical administration Administration regimens}

Staloral 300 contains equal proportions of D. pteronyssinus and $D$. farinae and is available as a minipump-predosed actuator. Similar to other allergen extracts, sublingual immunotherapy traditionally involves an uptitration phase followed by maintenance treatment at the maximal dose. The recommended doses during the buildup phase, with a daily dosing interval, are 1-2-4-8-10-30-60-120-240 IR. The 120 IR or 240 IR dose, depending on individual tolerability, is then used for maintenance treatment 3-7 times a week. Rush and ultrarush inductions have also been proposed for routine use, based on the favorable safety profile of Staloral, including for children under the age of 5 years. ${ }^{27}$ Ultrarush schemes with a buildup shorter than 2 hours have been reported in adults ${ }^{28}$ and children, ${ }^{29,30}$ with satisfactory results. In the study in adults, the buildup ultrarush phase involved administration every 5 minutes of increasing doses, reaching a cumulative allergen extract solution after 30 minutes that was several times the dose administered at the start of subcutaneous immunotherapy (range 4.7-525 $\mu \mathrm{g}$ of major allergens); all patients tolerated the treatment well. ${ }^{28}$ In the study in children, the ultrarush buildup phase involved administration every 10 minutes of increasing doses of the highest concentration vial of sublingual immunotherapy, with mild side effects (mainly oral symptoms) in $19 \%$ of patients. ${ }^{29}$

The allergen extract is usually taken in the morning before breakfast, is kept under the tongue for a few minutes, and then swallowed. The term sublingual immunotherapy, unless otherwise stated, indicates the sublingual swallow modality. The maintenance dose is generally the same for all patients, based on the fact that, unlike subcutaneous immunotherapy, administration of very large amounts of allergens by the sublingual route does not provoke severe side effects. ${ }^{31}$ In one study, maintenance treatment based on a continuous or intermittent schedule, ie, with alternate periods of 2-month treatment and 2-month interruption, was equally safe. ${ }^{32}$

\section{Criteria for starting and stopping treatment}

Due to the perennial nature of exposure to dust mites, Staloral 300 (Stallergenes, Antony, France) can be started at any period of the year. The duration of the treatment must be at least 3 years, as suggested in the position paper on sublingual immunotherapy from the World Allergy Organization in $2009 .{ }^{33}$ This duration is usually sufficient in most patients, but if the clinical symptoms of allergy are not adequately controlled, it is possible to prolong sublingual immunotherapy for up to 5 years. To continue treatment beyond this time is unwarranted.

\section{Clinical efficacy}

There has been a number of double-blind, placebo-controlled studies on the efficacy of sublingual immunotherapy using dust mite extract in patients with mite-induced allergic rhinitis and allergic asthma. In 2009, Compalati et al performed a meta-analysis according to the Cochrane recommendations, as described by Jorgensen et al. ${ }^{34}$ Globally, 12 studies fulfilled the selection criteria, eight evaluating allergic rhinitis and nine evaluating allergic asthma (some studies investigated both diseases). The eight studies of allergic rhinitis included 194 actively treated patients and 188 patients receiving placebo, and a significant reduction of symptoms $(P=0.02)$ and rescue medications $(P=0.04)$ was found. The nine studies of allergic asthma included 243 actively treated patients and 209 patients receiving placebo, and a significant reduction of symptoms $(P=0.02)$ and rescue medications $(P=0.02)$ was found. The authors concluded that there was promising evidence of efficacy for sublingual immunotherapy using mite extract, but suggested a need for more data from large, highquality, population-based studies. ${ }^{35}$ Of note, of the 12 studies included in the meta-analysis, six $^{36-41}$ used Staloral mite, and five used Staloral 300. ${ }^{36,37,39-41}$ The main characteristics of Staloral 300 are reported in Table 1. A placebo-controlled study is also available addressing the efficacy of Staloral 300 in mite-induced perennial conjunctivitis. In a group of 45 allergic patients, 26 actively treated patients showed a significant increase $(P<0.04)$ in the allergen dose giving

Table I Main characteristics of Staloral 300

\begin{tabular}{ll}
\hline Culture medium & Stalmite APF \\
Biological standardization & Index of reactivity \\
Der P I major allergen content & $60 \mu \mathrm{g} / 300 \mathrm{IR}$ \\
Der P 2 major allergen content & $\mathrm{I} 2 \mu \mathrm{g} / 300 \mathrm{IR}$ \\
Der f I major allergen content & $\mathrm{I} 50 \mu \mathrm{g} / 300 \mathrm{IR}$ \\
EBM recommendation based on randomized, & Asthma: A \\
placebo-controlled trials & Rhinitis: A \\
& Conjunctivitis: A \\
\hline
\end{tabular}

Abbreviations: APF, animal protein-free; EBM, evidence-based medicine; IR, index of reactivity. 
a positive conjunctival provocation test compared with 19 placebo-treated patients. ${ }^{42}$

Recent papers have evaluated the efficacy of Staloral 300 in real-life settings. In a group of 39 children with mite-induced rhinitis and asthma not controlled by mite avoidance measures, sublingual immunotherapy was able to reduce the mean number of asthma attacks per year from $8.2 \pm 3.05$ before treatment to $0.44 \pm 0.79$ after 3 years, ie, the recommended duration of sublingual immunotherapy; complete clinical remission was recorded in $95 \%$ of patients with asthma and in $82 \%$ of patients with rhinitis. ${ }^{43}$ In another study, 78 children with mite-induced rhinitis were evaluated before initiation of sublingual immunotherapy and after 6 , $12,24,36$, and 48 months of treatment. Patient evaluation of allergy severity using a visual analog scale revealed significant improvement $(P<0.001)$ after 6 months of treatment, which was maintained throughout the study period. The use of medications was also significantly reduced $(P<0.001)$ after 6 months and remained low until the end of the study. ${ }^{30}$

\section{Safety and tolerability}

The most common side effect of sublingual immunotherapy is the local reaction in the mouth, that is comparable with the local reaction at the site of injection of subcutaneous immunotherapy. The systematic revisions on global aspects of sublingual immunotherapy ${ }^{44}$ as well as on its safety ${ }^{31}$ did not identify any anaphylactic reactions in the trials regardless of the allergen extract used. A case of anaphylaxis to Staloral was described by Blazowski concerning a girl who, after maintenance treatment with a 10-drop dose of the extract, following an interruption of 3 weeks, ingested 60 drops of the extract and developed generalized urticaria, dyspnea, and hypotension..$^{45}$ This report highlights that even when using a safe method of administration such as the sublingual route, very high doses of allergen extracts are able to elicit severe systemic reactions. As far as local reactions are concerned, such as oral itching and burning or gastrointestinal symptoms once the extract is swallowed, proper management based on dose adjustment allows a maintenance dose to be reached. ${ }^{46}$ It is of interest that in the systematic revision on safety of sublingual immunotherapy, no relationship between dose and systemic reactions was found, whereas local reactions were more frequent during treatment with low-dose extracts. ${ }^{31}$

\section{Cost-effectiveness}

The potential of allergen immunotherapy to reduce health care expenditure in patients with respiratory allergy can be understood by considering the reduction in drug use reported in consensus documents and meta-analyses. ${ }^{33,35}$ Moreover, immunotherapy extends its clinical efficacy beyond the period of administration, because its mechanism of action modifies the natural history of the allergy, and thus amplifies the savings in medical resources. The economic advantages of allergen immunotherapy over drug therapy for respiratory allergy, as evaluated in specifically designed studies, was first reported in $1995^{47}$ and the available literature was recently reviewed by Berto et al. ${ }^{48}$ This review showed that the economic studies of allergic rhinitis and asthma focus on three main areas, ie, studies aimed at determining the cost of illness and studies focused either on a simple cost comparison amongst available therapeutic alternatives or directly comparing alternatives using full economic evaluation, such as cost-effectiveness and cost utility measures. These studies have shown that immunotherapy may be very beneficial to health care systems, because it can achieve a satisfactory clinical outcome at a reduced cost versus standard therapy alone, and may accrue extra benefit at an acceptable additional cost. This is also acceptable from a societal perspective, ie, when indirect costs of lost productivity are considered and included in the economic analysis. A study using Staloral 300 is available among the published studies on cost-effectiveness of immunotherapy. ${ }^{49}$ This study included 70 patients with mite-induced asthma, comprising 50 treated with sublingual immunotherapy in addition to drugs as needed and 20 treated with drugs alone. The patients were evaluated during a 3 -year course of sublingual immunotherapy and for 2 further years after stopping treatment. The patients treated with sublingual immunotherapy in addition to drug therapy had a higher mean annual cost compared with patients treated with drug therapy alone only in the first year of treatment; there was no difference at year 2, the cost was lower (but not significantly so) at year 3 for patients treated with sublingual immunotherapy, and, at years 4 and 5, the cost became significantly lower after stopping sublingual immunotherapy. The total cost at year 5 was 3881 Euros for patients treated with sublingual immunotherapy in addition to drugs compared with 5020 Euros for those treated with drugs alone, representing a mean saving of $23 \%$. Savings increased with disease severity, reaching $34 \%$ in patients with severe asthma.

\section{Conclusion}

The dust mite extract, Staloral 300, has good evidence for clinical efficacy, safety, and cost-effectiveness in patients with mite-induced respiratory allergy. The double-blind, placebo controlled trials performed using this product 
have provided a considerable part of the scientific evidence obtained in a meta-analysis of the efficacy of allergen immunotherapy in mite-induced rhinitis and asthma. ${ }^{35}$ The safety of Staloral 300 relies upon the quality of the procedures used in its preparation, particularly the culture medium used for production of the extract, as well as the known low risk of adverse reactions with the sublingual route. Another advantage of sublingual immunotherapy over subcutaneous immunotherapy is patient compliance, given that compliance with the latter is limited by the inconvenience of frequent injections. ${ }^{50}$ Finally, the cost-effectiveness, showing a cost-saving capacity of immunotherapy in general and of sublingual immunotherapy in particular, ${ }^{48}$ is an essential consideration when allocating treatment for optimal management of mite-allergic patients. Allergen immunotherapy is the only treatment presently aimed at targeting the cause and not just the symptoms of respiratory allergy, but the quality of the materials to be used is crucial in achieving such an outcome.

\section{Expert opinion}

The treatments available for respiratory allergy include allergen avoidance, drug therapy, and allergen immunotherapy, but only allergen immunotherapy is able to address the cause and not just the symptoms of allergy. Allergen immunotherapy is the practice of administering gradually increasing doses of a specific causative allergen to reduce the clinical reactivity of allergic subjects. It was introduced a century ago ${ }^{51}$ but remained an empirical treatment until 1954, when the first controlled trial ${ }^{52}$ was performed, paving the way for a scientific approach to the treatment of allergy. Today, allergen immunotherapy may be administered in two forms, ie, subcutaneous immunotherapy and sublingual immunotherapy. A large number of trials, globally analyzed in several meta-analyses, ${ }^{35,53-56}$ have evaluated the efficacy and safety of subcutaneous immunotherapy and sublingual immunotherapy in patients with allergic rhinitis and asthma. The available data provide solid evidence of clinical efficacy for both routes of administration. The mechanism of action of allergen immunotherapy involves modification, via allergen capture and presentation by Langerhans-type antigen-presenting cells, of the phenotype of $\mathrm{T}$ cells, which in allergic subjects is characterized by a prevalence of the Th 2 type. The changes induced result in a Th1-type response (immune deviation) related to increased production of interferon- $\gamma$ and IL- 2 or to reduced Th2 activity, through a mechanism known as anergy or tolerance. It is now known that $\mathrm{T}$ cell tolerance is characterized by generation of allergen-specific regulatory T cells producing cytokines such as IL-10 and transforming growth factor-beta, which have immunosuppressant and/or immunoregulatory activity. These mechanisms are similar in subcutaneous immunotherapy $y^{57,58}$ and sublingual immunotherapy. ${ }^{59}$

The house dust mite is the major cause of respiratory allergy worldwide, and allergen immunotherapy is an important part of the strategy to control this mite-induced disease.$^{60}$ The efficacy of subcutaneous immunotherapy in mite-induced asthma has been clearly demonstrated, ${ }^{53,56}$ but possible systemic reactions to injection of the allergen extract is a drawback. A risk factor for systemic reactions is the presence of asthma when the extract is administered, and this is particularly frequent in patients with mite allergy who are continuously exposed to a specific allergen. Reports in the 1980s of fatal anaphylactic reactions to subcutaneous immunotherapy ${ }^{61,62}$ highlighted this important issue, and have led to decreased use of this form of immunotherapy, especially after sublingual immunotherapy was introduced. In the conclusion of the latest meta-analysis by Abramson et al on subcutaneous immunotherapy in asthma, it was stated that the possibility of local or systemic adverse effects (such as anaphylaxis) must be considered in any patient. ${ }^{56}$ Sublingual immunotherapy, and particularly Staloral 300 , has shown a reassuring safety profile. Only one anaphylactic reaction has been reported as a consequence of erroneous ingestion of a huge dose of the allergen extract, ${ }^{45}$ and the usual safety and tolerability profile of sublingual immunotherapy enables administration of the high doses needed for clinical efficacy, immunological changes, ${ }^{63}$ and long-term disease-modifying effects.

\section{Disclosure}

Franco Frati, Simona Seta, Guglielmo Padua, Eleonora Cattaneo, Ilaria Dell'Albani, and Paola Puccinelli are employees of Stallergenes Italy. Marie David and Silvia Scurati are employees of Stallergenes France. Cristoforo Incorvaia is a scientific consultant of Stallergenes Italy.

\section{References}

1. Platts-Mills TAE, De Weck AL. Dust mite allergens and asthma - a worldwide problem. J Allergy Clin Immunol. 1989;83:416-427.

2. Arlian LG, Morgan MS, Neal JS. Dust mite allergens: ecology and distribution. Curr Allergy Asthma Rep. 2002;2:401-411.

3. Zock JP, Heinrich J, Jarvis D, et al. Distribution and determinants of house dust mite allergens in Europe: the European Community Respiratory Health Survey II. J Allergy Clin Immunol. 2006;118: 682-690.

4. Colloff MJ. Taxonomy and identification of dust mites. Allergy. 1998; 53 Suppl 48:7-12. 
5. Thomas WR, Hales BJ, Smith WA. House dust mite allergens in asthma and allergy. Trends Mol Med. 2010;16:321-328.

6. O'Neil SE, Heinrich TK, Hales BJ, et al. The chitinase allergens Der p 15 and Der p 18 from Dermatophagoides pteronyssinus. Clin Exp Allergy. 2006;36:831-839.

7. Thomas WR, Heinrich TK, Smith WA, Hales BJ. Pyroglyphid house dust mite allergens. Protein Pept Lett. 2007;14:943-953.

8. Park GM, Lee SM, Lee IY, et al. Localization of a major allergen, Der $\mathrm{p} 2$, in the gut and faecal pellets of Dermatophagoides pteronyssinus. Clin Exp Allergy. 2000;30:1293-1297.

9. Pittner G, Vrtala S, Thomas WR, et al. Component-resolved diagnosis of house dust mite allergy with purified natural and recombinant mite allergens. Clin Exp Allergy. 2004;34:597-603.

10. Reese G, Ayuso R, Lehrer SB. Tropomyosin: an invertebrate pan-allergen. Int Arch Allergy Immunol. 1999;119:247-258.

11. Hales BJ, Martin AC, Pearce LJ, et al. IgE and IgG anti-house dust mite specificities in allergic disease. J Allergy Clin Immunol. 2006;118:361-367.

12. Kidon MI, Chin CW, Kang LW, et al. Mite component-specific IgE repertoire and phenotypes of allergic disease in childhood: the tropical perspective. Pediatr Allergy Immunol. 2011;22:202-210.

13. Karp CL. Guilt by intimate association: what makes an allergen an allergen? J Allergy Clin Immunol. 2010;125:955-960.

14. Jacquet A. The role of house dust mites-induced innate immunity in development of allergic response. Int Arch Allergy Immunol. 2011;155:95-105.

15. Bessot JC, Pauli G. Mite allergens: an overview. Eur Ann Allergy Clin Immunol. 2011;43:141-156.

16. Jacquet $A$. The role of innate immunity activation in house dust mite allergy. Trends Mol Med. 2011;17:604-611.

17. Novak N, Koch S, Allam JP, Bieber T. Dendritic cells: bridging innate and adaptive immunity in atopic dermatitis. J Allergy Clin Immunol. 2010;125:50-59.

18. Inal A, Kendirli SG, Yilmaz M, et al. Indices of lower airway inflammation in children monosensitized to house dust mite after nasal allergen challenge. Allergy. 2008;63:1345-1351.

19. Incorvaia C, Frati F, Verna N, D'Alò S, Motolese A, Pucci S. Allergy and the skin. Clin Exp Immunol. 2008;153 Suppl 1:27-29.

20. Leung DYM. Our evolving understanding of the functional role of filaggrin in atopic dermatitis. J Allergy Clin Immunol. 2009;124: 494-495.

21. Guilbert TW, Morgan WJ, Zeiger RS, et al. Long-term inhaled corticosteroids in preschool children at high risk of asthma. $N$ Engl J Med. 2006;354:1985-1997.

22. Simpson A, Custovic A. The role of allergen avoidance in the secondary prevention of atopic disorders. Curr Opin Allergy Clin Immunol. 2005;5:223-227.

23. Incorvaia $C$, Frati F. One century of allergen-specific immunotherapy for respiratory allergy. Immunotherapy. 2011;3:629-635.

24. Dreborg S. Mite allergens: collection, determination, expression of results and risk levels for sensitization and symptoms induction. Allergy. 1998;53 Suppl 48:88-91.

25. Batard T, Hrabina A, Bi XZ, et al. Production and proteomic characterization of pharmaceutical-grade Dermatophagoides pteronyssinus and Dermatophagoides farinae extracts for allergy vaccines. Int Arch Allergy Immunol. 2006;140:295-305.

26. van Ree R. Indoor allergens: relevance of major allergen measurements and standardization. J Allergy Clin Immunol. 2007;119:270-277.

27. Fiocchi A, Pajno G, La Grutta S, et al. Safety of SLIT in children aged 3 to 7 years. Ann Allergy Asthma Immunol. 2005;95:254-258.

28. Rossi R, Monasterolo RE. A pilot study of feasibility of ultra-rush (20-25 minutes) sublingual-swallow immunotherapy in 679 patients (699 sessions) with allergic rhinitis and/or asthma. Int J Immunopathol Pharmacol. 2005;18:277-285.

29. Tripodi S, Di Rienzo Businco A, Benincori N, et al. Safety and tolerability of ultra-rush induction, less than one hour, of sublingual immunotherapy in children. Int Arch Allergy Immunol. 2005;139:149-152.
30. Ferres J, Justicia JR, Garcia MP, et al. Efficacy of high-dose sublingual immunotherapy in children allergic to house dust mites in real-life clinical practice. Allergol Immunopathol (Madr). 2011;39:122-127.

31. Gidaro GB, Marcucci F, Sensi L, et al. The safety of sublingual-swallow immunotherapy: an analysis of published studies. Clin Exp Allergy. 2005;35:565-571.

32. Cadario G, Ciprandi G, Di Cara G, et al. Comparison between continuous or intermittent schedules of sublingual immunotherapy for house dust mites: effects on compliance, patient's safisfaction, quality of life, and safety. Int J Immunopathol Pharmacol. 2008;21:471-473.

33. Canonica GW, Bousquet J, Casale T, et al. Sublingual immunotherapy: World Allergy Organization Position Paper 2009. Allergy. 2009; 64 Suppl 91:1-59.

34. Jorgensen AW, Hilden J, Gotzsche PC. Cochrane reviews compared with industry supported meta-analyses and other meta-analyses of the same drugs: systematic review. Br Med J. 2006;333:782.

35. Compalati E, Passalacqua G, Bonini M, Canonica GW. The efficacy of sublingual immunotherapy for house dust mites respiratory allergy: results of a GA2LEN meta-analysis. Allergy. 2009;64:1570-1579.

36. Bousquet J, Scheinmann P, Guinnepain MT, et al. Sublingual swallow immunotherapy (SLIT) in patients with asthma due to house dust mites: a double-blind placebo-controlled study. Allergy. 1999;54:249-260.

37. Guez S, Vatrinet $\mathrm{C}$, Fadel R, Andrè $\mathrm{C}$. House dust mite sublingual swallow immunotherapy in perennial rhinitis: a double-blind placebocontrolled study. Allergy. 2000;55:369-375.

38. Bahcecilier NN, Isik U, Barlan IB, Basaran N. Efficacy of sublingual immunotherapy in children with asthma and rhinitis: a double-blind, placebo-controlled trial. Pediatr Pulmonol. 2001;32:49-55.

39. Niu CK, Chen WY, Huang JL, et al. Efficacy of sublingual immunotherapy with high-dose mite extracts in asthma: a multi-center, double-blind, randomized, and placebo-controlled study in Taiwan. Respir Med. 2006;100:1374-1383.

40. Lue KH, Lin YH, Sun HL, et al. Clinical and immunological effect of sublingual immunotherapy in asthmatic children sensitized to mites: a double-blind, randomized, placebo-controlled study. Pediatr Allergy Immunol. 2006;17:408-415.

41. Pham-Ti N, Scheinmann P, Fadel R, et al. Assessment of sublingual immunotherapy efficacy in children with house dust mite-induced allergic asthma optimally controlled by pharmacologic treatment and mite-avoidance measures. Pediatr Allergy Immunol. 2007;18:47-57.

42. Mortemousque B, Bertel F, De Casamayor P, et al. House-dust mite sublingual-swallow immunotherapy in perennial conjunctivitis: a double-blind, placebo-controlled study. Clin Exp Allergy. 2003:33464-33469.

43. Nuhoglu Y, Ozumut SS, Ozdemir C, et al. Sublingual immunotherapy to house dust mites in pediatric patients with allergic rhinitis and asthma: a retrospective analysis of clinical course over a 3-year follow-up period. J Investig Allergol Clin Immunol. 2007;17:375-378.

44. Radulovic S, Wilson D, Calderon M, Durham S. Systematic reviews of sublingual immunotherapy (SLIT). Allergy. 2011;66:740-752.

45. Blazowski L. Anaphylactic shock because of sublingual immunotherapy overdose during third year of maintenance dose. Allergy. 2008;63:374.

46. Frati F, Sensi L, Di Rienzo V, et al. A model for management of sublingual immunotherapy. Eur Ann Allergy Clin Immunol. 2003;35:56-60.

47. Buchner K, Siepe M. Nutzen der Hyposensibilisierung unter wirtschaftlichen Aspekten. [Benefits of immunotherapy with economic aspects]. Allergo J. 1995;4:156-163. German.

48. Berto P, Frati F, Incorvaia C. Economic studies of immunotherapy: a review. Curr Opin Allergy Clin Immunol. 2008;8:585-589.

49. Ariano R, Berto P, Incorvaia C, et al. Economic evaluation of sublingual immunotherapy vs symptomatic treatment in allergic asthma. Ann Allergy Asthma Immunol. 2009;103:254-259.

50. Incorvaia C, Mauro M, Ridolo E, et al. Patient's compliance with allergen immunotherapy. Patient Prefer Adherence. 2008;2:247-261.

51. Noon L, Cantab BC, Eng FR. Prophylactic inoculation against hay fever. Lancet. 1911;177:1572-1573. 
52. Frankland A, Augustin R. Prophylaxis of summer hay fever and asthma: a controlled trial comparing crude grass pollen extract with the isolated main protein components. Lancet. 1954;266:1055-1058.

53. Abramson MJ, Puy RM, Weiner JM. Is allergen immunotherapy effective in asthma? A meta-analysis of randomized controlled trials. Am J Respir Crit Care Med. 1995;151:969-974.

54. Calderon MA, Alves B, Jacobson M, et al. Allergen injection immunotherapy for seasonal allergic rhinitis. Cochrane Database Syst Rev. 2007;1:CD001936.

55. Radulovic S, Calderon MA, Wilson D, Durham S. Sublingual immunotherapy for allergic rhinitis. Cochrane Database Syst Rev. 2010;12:CD002893.

56. Abramson MJ, Puy RM, Weiner JM. Injection immunotherapy for asthma. Cochrane Database Syst Rev. 2010;8:CD001186.

57. Maggi E. T cell responses induced by allergen-specific immunotherapy Clin Exp Immunol. 2010;161:10-18.
58. Taylor A, Verhagen J, Blaser K, et al. Mechanisms of immune suppression by interleukin-10 and transforming growth factor beta: the role of T regulatory cells. Immunology. 2006;117:433-442.

59. Bohle B, Kinaciyan T, Gestrmayr M, et al. Sublingual immunotherapy induces IL-10-producing T regulatory cells, allergen-specific T. cell tolerance, and immune deviation. J Allergy Clin Immunol. 2007; 120:707-713.

60. Gaffin JM, Phipatanakul W. The role of indoor allergens in the development of asthma. Curr Opin Allergy Clin Immunol. 2009;9:128-135.

61. Committee on Safety in Medicine. CSM update: desensitizing vaccines Br Med J. 1986;293:948.

62. Lockey RF, Benedikt LM, Turkeltaub PC, Bukantz SC. Fatalities from immunotherapy (IT) and skin testing (ST). J Allergy Clin Immunol. 1987;79:660-677.

63. Marcucci F, Sensi L, Di Cara G, et al. Dose dependence of immunological response to sublingual immunotherapy. Allergy. 2005;60:952-956.

\section{Publish your work in this journal}

Drug Design, Development and Therapy is an international, peerreviewed open-access journal that spans the spectrum of drug design and development through to clinical applications. Clinical outcomes, patient safety, and programs for the development and effective, safe, and sustained use of medicines are a feature of the journal, which has also been accepted for indexing on PubMed Central. The manuscript management system is completely online and includes a very quick and fair peer-review system, which is all easy to use. Visit http://www.dovepress.com/testimonials.php to read real quotes from published authors

Submit your manuscript here: http://www.dovepress.com/drug-design-development-and-therapy-journal 